\title{
MODIFIED NASH TRIVIALITY OF A FAMILY OF ZERO-SETS OF WEIGHTED HOMOGENEOUS POLYNOMIAL MAPPINGS
}

\author{
By SATOSHI KoIKe
}

\section{§0. Introduction.}

Let $\alpha=\left(\alpha_{1}, \cdots, \alpha_{n}\right)$ be an $n$-tuple of positive integers. Assume that the greatest common divisor of $\alpha_{j}$ 's is 1 . Let $\mathbf{N}$ denote the set of positive integers, and let $\mathbf{R}$ denote the set of real numbers. Let $f:\left(\mathbf{R}^{n}, 0\right) \rightarrow(\mathbf{R}, 0)$ be a polynomial function defined by

$$
f(x)=\sum_{\beta} A_{\beta} x_{1}^{\beta_{1}} \cdots x_{n}^{\beta_{n}} \quad\left(A_{\beta} \neq 0, \beta_{1}, \cdots, \beta_{n} \in \mathbf{N} \cup\{\mathbf{0}\}\right) .
$$

We say that $f$ is werghted homogeneous of type $\left(\alpha_{1}, \cdots, \alpha_{n} ; L\right)\left(\alpha_{1}, \cdots, \alpha_{n}, L \in \mathbf{N}\right)$, if

$$
\alpha_{1} \beta_{1}+\cdots+\alpha_{n} \beta_{n}=L \text { for any } \beta=\left(\beta_{1}, \cdots, \beta_{n}\right) .
$$

Let $J$ be an open interval, and $t_{0} \in J$. Let $f_{t}:\left(\mathbf{R}^{n}, 0\right) \rightarrow\left(\mathbf{R}^{p}, 0\right)$ be a polynomial mapping where each $f_{t, 2}$ is weighted homogeneous of type $\left(\alpha_{1}, \cdots, \alpha_{n} ; L_{i}\right)(1 \leqq i \leqq p)$ for $t \in J$. We define a mapping $F:\left(\mathbf{R}^{n} \times J,\{0\} \times J\right) \rightarrow\left(\mathbf{R}^{p}, 0\right)$ by $F(x, t)=f_{t}(x)$. Assume that $F$ is a polynomial mapping (or of class $C^{2}$ ). It is well-known that the following fact holds under these assumptions:

FACT. If $f_{t}^{-1}(0) \cap \sum f_{t}=\{0\}$ for any $t \in J$ (where $\sum f_{t}$ denotes the singular points set of $\left.f_{t}\right)$, then $\left(\mathbf{R}^{n} \times J, F^{-1}(0)\right)$ is topologically trivial i.e. there exists a t-level preserving homeomorphism $\sigma:\left(\mathbf{R}^{n} \times J,\{0\} \times J\right) \rightarrow\left(\mathbf{R}^{n} \times J,\{0\} \times J\right)$ such that

$$
\sigma\left(\left(R^{n} \times J, F^{-1}(0)\right)\right)=\left(\mathbf{R}^{n} \times J, f_{t_{0}}^{-1}(0) \times J\right)
$$

Remark 1. Results generalizing this fact have been obtained in [2], [5]. But it seems that the fact itself was recognized by many mathematicians a good while ago.

Since we consider the weighted homogeneous case with an isolated singularity, it seems natural that stronger triviality than topological one holds. In fact, such triviality called "modified Nash triviality" holds under the above assumptions (see Theorem in $\S 2$ ). On the other hand, we have introduced the notion of "strong $C^{0}$ triviality" for a family of analytic functions in [6]. Roughly speaking, strong $C^{0}$ equivalence is a $C^{0}$ equivalence which preserves the tangency of analytic arcs at $0 \in \mathbf{R}^{n}$. In $\S 4$, we discuss the relation between modified Nash triviality and strong $C^{0}$ triviality for a family of zero-sets of weighted homogeneous polynomials.

The author would like to thank Professor M. Shiota for helpful suggestions concerning Proposition 2 in $\S 3$ (semi-algebraic triviality theorem).

Received April 30, 1993. 


\section{§1. Some properties of Nash manifolds.}

In this section, we recall some important results on Nash manifolds. A semi-algebraic set of $\mathbf{R}^{n}$ is a finite union of sets of the form

$$
\left\{x \in \mathbf{R}^{n} \mid f_{1}(x)=\cdots=f_{k}(x)=0, g_{1}(x)>0, \cdots, g_{m}(x)>0\right\},
$$

where $f_{1}, \cdots, f_{k}, g_{1}, \cdots, g_{m}$ are polynomial functions on $\mathbf{R}^{n}$. Let $r=0,1,2, \cdots, \infty, \omega$. A semi-algebraic set of $\mathbf{R}^{n}$ is called a $C^{r}$ (affine) Nash manifold if it is a regular $C^{r}$ submanifold of $\mathbf{R}^{n}$. Let $M \subset \mathbf{R}^{m}$ and $N \subset \mathbf{R}^{n}$ be $C^{r}$ Nash manifolds. A $C^{s}$ mapping $f: M \rightarrow N(s \leqq r)$ is called a $C^{s}$ Nash mapping if the graph of $f$ is semi-algebraic in $\mathbf{R}^{m} \times \mathbf{R}^{n}$. fold.

Theorem 1 (B. Malgrange [10]). (1) A $C^{\infty}$ Nash manifold ıs a $C^{\omega}$ Nash manı-

(2) A $C^{\infty}$ Nash mappıng between $C^{\omega}$ Nash manifolds is a $C^{\omega}$ Nash mapping.

After this, a Nash manifold and a Nash mapping mean a $C^{\omega}$ Nash manifold and a $C^{\omega}$ Nash mapping, respectively.

THEOREM 2 (M. Shiota [11]). Let $M_{1} \supset N_{1}, M_{2} \supset N_{2}$ be compact Nash manifolds and compact Nash submanifolds. If the pairs $\left(M_{1}, N_{1}\right)$ and $\left(M_{2}, N_{2}\right)$ are $C^{\infty}$ diffeomorphic, then they are Nash diffeomorphıc.

Remark 2. In Theorem 2, we can replace the assumption of " $C^{\infty}$ diffeomorphic" by " $C^{1}$ diffeomorphic" ([12]).

THEOREM 3 (M. Shiota [12]). There exist two (affine) Nash manifolds which are $C^{\omega}$ diffeomorphic but not Nash diffeomorphıc.

In general, Nash diffeomorphism is stronger than the notion of $C^{\omega}$ diffeomorphism.

\section{§2. Result.}

Let $\alpha=\left(\dot{\alpha_{1}}, \cdots, \alpha_{n}\right)$ be an $n$-tuple of positive integers. Put $\rho=\alpha_{1} \cdots \alpha_{n}$ and $\rho_{i}=\rho / \alpha_{i}(1 \leqq i \leqq n)$. Set

$$
S_{1}(\alpha)=\left\{\left(X_{1}, \cdots, X_{n}\right) \in \mathbf{R}^{n} \mid X_{1}^{2 \rho_{1}}+\cdots+X_{n}^{2 \rho_{n}}=1\right\} .
$$

We define $\pi_{\alpha}: S_{1}(\alpha) \times \mathbf{R} \rightarrow \mathbf{R}^{n}$ by

$$
\pi_{\alpha}\left(X_{1}, \cdots, X_{n} ; u\right)=\left(u^{\alpha_{1}} X_{1}, \cdots, u^{\alpha_{n}} X_{n}\right) .
$$

Put $E=S_{1}(\alpha) \times \mathbf{R}$ and $E_{0}=\pi_{\alpha}^{-1}(0)=S_{1}(\alpha) \times\{0\}$. Then $E$ is a Nash manifold and $E_{0}$ is a Nash submanifold. The restricted mapping $\left.\pi_{\alpha}\right|_{E-E_{0}} \cdot E-E_{0} \rightarrow \mathbf{R}^{n}-\{0\}$ is a $2: 1$ mapping. Therefore $\pi_{\alpha}:\left(E, E_{0}\right) \rightarrow\left(\mathbf{R}^{n}, 0\right)$ is a finite Nash modification. Let $J$ be an open interval and $t_{0} \in J$, and let $f_{t}:\left(\mathbf{R}^{n}, 0\right) \rightarrow\left(\mathbf{R}^{p}, 0\right)(t \in J)$ be a weighted homogeneous polynomial mapping. We define $F:\left(\mathbf{R}^{n} \times J,\{0\} \times J\right) \rightarrow\left(\mathbf{R}^{p}, 0\right)$ by $F(x, t)=f_{t}(x)$.

Definition. We say that $\left(\mathbf{R}^{n} \times J, F^{-1}(0)\right)$ admits a $\pi_{\alpha}$-modified Nash trivial- 
ızation, if there exists a $t$-level preserving Nash diffeomorphism $\Phi:\left(E \times J, E_{0} \times J\right) \rightarrow$ $\left(E \times J, E_{0} \times J\right)$ which induces a $t$-level preserving homeomorphism $\phi:\left(\mathbf{R}^{n} \times J,\{0\} \times J\right) \rightarrow$ $\left(\mathbf{R}^{n} \times J,\{0\} \times J\right)$ such that

$$
\phi\left(\left(\mathbf{R}^{n} \times J, F^{-1}(0)\right)\right)=\left(\mathbf{R}^{n} \times J, f_{t_{0}}^{-1}(0) \times J\right) .
$$

THEOREM. Let $J$ be an open interval, and let $f_{t}:\left(\mathbf{R}^{n}, 0\right) \rightarrow\left(\mathbf{R}^{p}, 0\right)$ be a polynomial mapping where each $f_{t, \imath}$ is werghted homogeneous of type $\left(\alpha_{1}, \cdots, \alpha_{n} ; L_{i}\right)(1 \leqq i \leqq p)$ for $t \in J$. Assume that $F:\left(\mathbf{R}^{n} \times J,\{0\} \times J\right) \rightarrow\left(\mathbf{R}^{p}, 0\right)$ is a polynomial mapping. If $f_{t}^{-1}(0) \cap \sum f_{t}=\{0\}$ for any $t \in J$, then $\left(\mathbf{R}^{n} \times J, F^{-1}(0)\right)$ admits a $\pi_{\alpha}$-modified Nash trivialization.

Remark 3. In the case $n \leqq p, f_{t}^{-1}(0) \cap \sum f_{t}=\{0\}$ implies $f_{t}^{-1}(0)=\{0\}$

Example 1. Let $f_{t}:\left(\mathbf{R}^{3}, 0\right) \rightarrow(\mathbf{R}, 0)(t \in \mathbf{R})$ be a weighted homogeneous polynomial of type $\alpha=(1,2,3 ; 13)$ defined by

$$
f_{t}(x, y, z)=x^{13}+x y^{6}+x z^{4}+t y^{5} z .
$$

Then $\frac{\partial f_{t}}{\partial x}=13 x^{12}+y^{6}+z^{4}$. Therefore each $f_{t}$ has an isolated singularity. It follows from the Theorem that $\left(\mathbf{R}^{3} \times \mathbf{R}, F^{-1}(0)\right)$ admits a $\pi_{\alpha}$-modified Nash trivialization.

Problem 1. Several kinds of topological triviality theorems for a family of analytic varieties are known. Do modified Nash triviality theorems hold under the same assumption for a family of algebraıc varietıes?

\section{§3. Outline of the proof of the theorem.}

The proof of the Theorem consists of three parts.

STEP 1. Remark that $S_{1}(\alpha) \cap f_{t}^{-1}(0)(t \in J)$ and $S_{1}(\alpha) \times J \cap F^{-1}(0)$ are Nash submanifolds of $S_{1}(\alpha)$ and $S_{1}(\alpha) \times J$, respectively.

Proposition 1. Under the same assumption as the Theorem, there exists a t-level preserving $C^{\infty}$ diffeomorphism

$$
H: S_{1}(\alpha) \times J \cap F^{-1}(0) \rightarrow\left(S_{1}(\alpha) \cap f_{t_{0}}^{-1}(0)\right) \times J .
$$

EuleR's Theorem. If $f:\left(\mathbf{R}^{n}, 0\right) \rightarrow(\mathbf{R}, 0)$ is werghted homogeneous of type $\left(\alpha_{1}\right.$, $\left.\cdots, \alpha_{n} ; L\right)$, then

$$
\alpha_{1} x_{1} \frac{\partial f}{\partial x_{1}}+\cdots+\alpha_{n} x_{n} \frac{\partial f}{\partial x_{n}}=L f
$$

Many singularists would know that such a $t$-level preserving $C^{\infty}$ diffeomorphism in Proposition 1 exists. But, thanks to Euler's Theorem, we can concretely construct a $C^{\infty}$ vector field on $S_{1}(\alpha) \times J \cap F^{-1}(0)$, called the Kuo vector field ([8], [9]), whose flow gives the diffeomorphism.

STEP 2. (semi-algebraic triviality theorem) 
Proposition 2. Let $M \supset N$ be a Nash manifold and a Nash (regular) submanifold such that $N$ is closed in $M$. Let $p: M \rightarrow \mathbf{R}$ be a proper Nash submersion such that the restriction of $p$ to $N$ is also a proper Nash submersion. Then there exists a Nash diffeomorphism

$$
\Phi:(M, N) \rightarrow\left(M_{0}, N_{0}\right) \times \mathbf{R}
$$

such that $p \circ \Phi^{-1}$ is the canonical projection onto $\mathbf{R}$, namely, $p \circ \Phi^{-1}\left(m_{0}, t\right)=t$ for $\left(m_{0}, t\right) \in M_{0} \times \mathbf{R}$, where $M_{0}=p^{-1}(0)$ and $N_{0}=\left(\left.p\right|_{N}\right)^{-1}(0)$.

Remark 4. We can replace $\mathbf{R}$ by an open interval $J$ in Proposition 2 .

We can prove this proposition, by using the following results :

(i) Proposition 2 holds in the case where $N=\emptyset$ ([3] Theorem 1).

(ii) For $0<r<\infty$, Proposition 2 holds in the case where $M$ is a $C^{r}$ Nash manifold with boundary $N$ ([3] Theorem 3$)$.

(iii) $C^{r}$ Nash tubular neighbourhood theorem holds for $r>1$ ([12] Lemma I.3.2).

(iv) $C^{r}$ Nash partition of unity holds for $0<r<\infty$ ([12] Corollaries II.2.8, 2.9 and Remark II.2.13).

(v) $C^{r}$ Nash approximation theorem holds for $r \leqq \omega$ ([12] Corollary II.5.7).

STEP 3. By Propositions 1 and 2, there exists a $t$-level preserving Nash diffeomorphism $G: S_{1}(\alpha) \times J \rightarrow S_{1}(\alpha) \times J$ such that

$$
G\left(S_{1}(\alpha) \times J \cap F^{-1}(0)\right)=\left(S_{1}(\alpha) \cap f_{t_{0}}^{-1}(0)\right) \times J .
$$

We write $G(x, t)=\left(\sigma_{t}(x), t\right)$ for $(x, t) \in S_{1}(\alpha) \times J$. We define a mapping $\Phi:\left(E \times J, E_{0} \times\right.$ $J) \rightarrow\left(E \times J, E_{0} \times J\right)$ by

$$
\Phi((x ; u), t)=\left(\left(\sigma_{t}(x) ; u\right), t\right) .
$$

Then this $\Phi$ gives the modified Nash trivialization in the Theorem.

\section{§4. Strong $C^{0}$ equivalence.}

First, we define the notion of strong $C^{0}$ equivalence.

Notation. (1) By an analytic arc at $0 \in \mathbf{R}^{n}$, we mean the germ of an analytic map $\lambda \cdot[0, \varepsilon) \rightarrow \mathbf{R}^{n}$ with $\lambda(0)=0, \lambda(s) \neq 0, s>0$. The set of all such arcs is denoted by $A\left(\mathbf{R}^{n}, 0\right)$.

(2) For $\lambda, \mu \in A\left(\mathbf{R}^{n}, 0\right), O(\lambda, \mu)>1$ (resp. $O(\lambda, \mu)=1$ ) means that arcs $\lambda, \mu$ are tangent (resp. crossing without touching) at $0 \in \mathbf{R}^{n}$.

Let $E_{|\omega|}(n, 1)$ be the set of analytic function germs : $\left(\mathbf{R}^{n}, 0\right) \rightarrow(\mathbf{R}, 0)$, and let $S\left(\mathbf{R}^{n}, 0\right)$ be the set of set germs at $0 \in \mathbf{R}^{n}$.

Definition. Given $f, g \in E_{|\omega|}(n, 1)$, we say that $\left(\mathbf{R}^{n}, f^{-1}(0)\right),\left(\mathbf{R}^{n}, g^{-1}(0)\right) \in$ $S\left(\mathbf{R}^{n}, 0\right)$ are strongly $C^{0}$ equivalent, if there exists a local homeomorphism $\sigma:\left(\mathbf{R}^{n}, 0\right) \rightarrow$ $\left(\mathbf{R}^{n}, 0\right)$ such that

(I) $\sigma\left(f^{-1}(0)\right)=g^{-1}(0)$, 
(II) if $\lambda \in A\left(\mathbf{R}^{n}, 0\right)$ with $\lambda \subset f^{-1}(0)$ (resp. $g^{-1}(0)$ ), then $\sigma(\lambda)$ (resp. $\left.\sigma^{-1}(\lambda)\right) \in$ $A\left(\mathbf{R}^{n}, 0\right)$, and

(III) for any $\lambda, \mu \in A\left(\mathbf{R}^{n}, 0\right)$ with $\lambda, \mu \subset f^{-1}(0), O(\lambda, \mu)=1$ if and only if $O(\sigma(\lambda)$, $\sigma(\mu))=1$.

Let $J$ be an open interval, and let $f_{t}:\left(\mathbf{R}^{n}, 0\right) \rightarrow(\mathbf{R}, 0)(t \in J)$ be a weighted homogeneous polynomial of type $\alpha=\left(\alpha_{1}, \cdots, \alpha_{n}\right)$ with an isolated singularity. In this section, we discuss the relation between $\pi_{\alpha}$-modified Nash triviality and strong $C^{0}$ triviality of the family $\left\{\left(\mathbf{R}^{n}, f_{t}^{-1}(0)\right)\right\}_{t \in J}$.

(A) Consider the homogeneous case i.e. $\alpha_{1}=\cdots=\alpha_{n}=1$. Recall the notations $E=S_{1}(\alpha) \times \mathbf{R}$ and $E_{0}=S_{1}(\alpha) \times\{0\}$. We say that $(X ; u)=\left(X_{1}, \cdots, X_{n} ; u\right),(Y ; s)=$ $\left(Y_{1}, \cdots, Y_{n} ; s\right) \in E$ are equivalent, if

(i) $X_{\imath}=Y_{\imath}(1 \leqq i \leqq n)$ and $u=s$, or

(ii) $X_{i}=-Y_{\imath}(1 \leqq i \leqq n)$ and $u=-s$.

Then this relation is an equivalence relation. We denote by $\widetilde{E}$ and $\widetilde{E_{0}}$ the quotient sets of $E$ and $E_{0}$ by the relation $\sim$, respectively. Let $\pi:\left(E, E_{0}\right) \rightarrow\left(\widetilde{E}, \widetilde{E_{0}}\right)$ be the quotient map, and let $\widetilde{\pi_{\alpha}}:\left(\widetilde{E}, \widetilde{E_{0}}\right) \rightarrow\left(\mathbf{R}^{n}, 0\right)$ be the blow up at $0 \in \mathbf{R}^{n}$. Then the following diagram commutes :

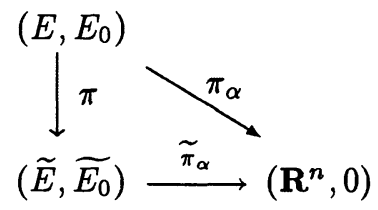

By the Theorem, $\left\{\left(\mathbf{R}^{n}, f_{t}^{-1}(0)\right)\right\}_{t \in J}$ admits a $\pi_{\alpha}$-modified Nash trivialization i.e. there exists a $t$-level preserving Nash diffeomorphism $\Phi:\left(E \times J, E_{0} \times J\right) \rightarrow\left(E \times J, E_{0} \times J\right)$ which induces a $t$-level preserving homeomorphism $\phi:\left(\mathbf{R}^{n} \times J,\{0\} \times J\right) \rightarrow\left(\mathbf{R}^{n} \times J,\{0\} \times\right.$ $J$ ) such that

$$
\phi\left(\left(\mathbf{R}^{n} \times J, F^{-1}(0)\right)\right)=\left(\mathbf{R}^{n} \times J, f_{t_{0}}^{-1}(0) \times J\right) \text { for } t_{0} \in J .
$$

In this case, the Nash diffeomorphism $\Phi$ induces a $t$-level preserving Nash diffeomorphism $\widetilde{\Phi}:\left(\widetilde{E} \times J, \widetilde{E_{0}} \times J\right) \rightarrow\left(\widetilde{E} \times J, E_{0} \times J\right)$ such that the following diagram commutes :

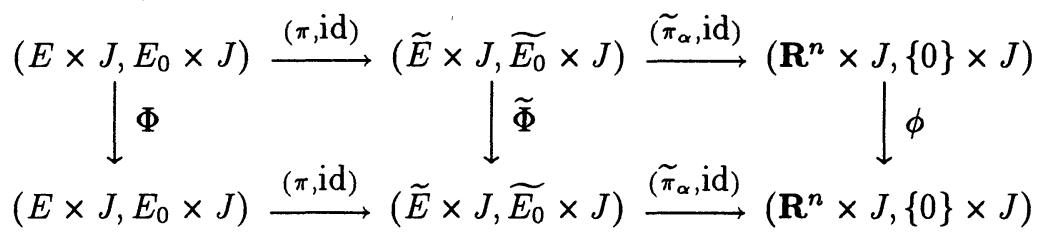

Therefore $\pi_{\alpha}$-modified Nash triviality implies strong $C^{0}$ triviality.

(B) Consider the case where $\alpha_{1}=\cdots=\alpha_{s}<\alpha_{s+1}=\cdots=\alpha_{n}(1 \leqq s<n)$.

Problem 2. Is the family $\left\{\left(\mathbf{R}^{n}, f_{t}^{-1}(0)\right)\right\}_{t \in J}$ strongly $C^{0}$ trivial?

The Theorem in $\S 2$ has come from this problem.

(C) Consider the case where $n=3$ and $\alpha_{1}<\alpha_{2}<\alpha_{3}$. 
Proposition 3. Let $f, g:\left(\mathbf{R}^{3}, 0\right) \rightarrow(\mathbf{R}, 0)$ be werghted homogeneous polynomials of type $\left(\alpha_{1}, \alpha_{2}, \alpha_{3} ; L\right)\left(\alpha_{1}<\alpha_{2}<\alpha_{3}\right)$ with an isolated singularity. Assume that two set germs $\left(\mathbf{R}^{3}, f^{-1}(0)\right),\left(\mathbf{R}^{3}, g^{-1}(0)\right) \in S\left(\mathbf{R}^{3}, 0\right)$ are strongly $C^{0}$ equivalent. If $f^{-1}(0) \supset$ $\left\{x_{1}=0\right\}$, then $g^{-1}(0) \supset\left\{x_{1}=0\right\}$.

Example 1. Let $f_{t}:\left(\mathbf{R}^{3}, 0\right) \rightarrow(\mathbf{R}, 0),(t \in \mathbf{R})$ be a weighted homogeneous polynomial defined in $\S 2$. Then, by Proposition $3,\left\{\left(\mathbf{R}^{3}, f_{t}^{-1}(0)\right)\right\}_{t \in \mathbf{R}}$ is not strongly $C^{0}$ trivial at $0 \in \mathbf{R}$.

Example 2 (Briançon-Speder family [1]). Let $f_{t}:\left(\mathbf{R}^{3}, 0\right) \rightarrow(\mathbf{R}, 0)$ be a weighted homogeneous polynomial defined by

$$
f_{t}(x, y, z)=z^{5}+t z y^{6}+y^{7} x+x^{15}
$$

for $|t|<1+\varepsilon$, where $\varepsilon$ is a sufficiently small positive number. Then each $f_{t}$ has an algebraically isolated singularity. But two set germs $\left(\mathbf{R}^{3}, f_{0}^{-1}(0)\right),\left(\mathbf{R}^{3}, f_{-1}^{-1}(0)\right) \in S\left(\mathbf{R}^{3}, 0\right)$ are not strongly $C^{0}$ equivalent (Theorem $\mathrm{A}$ in [6]).

It follows from the Theorem and the above examples that modified Nash triviality does not imply strong $C^{0}$ triviality in this case.

Remark 5. In [7], the author formulated a necessary condition for a family of weighted homogeneous polynomials of three variables to be strongly $C^{0}$ trivial. Recently T. Fukui has given a new approach to strong $C^{0}$ triviality of a family of polynomial functions of three variables, by using toric resolution ([4]).

\section{REFERENCE}

[1] J. Briançon and J.P. Speder, La trivialité topologique n'implique pas les conditions de Whitney, C.R. Acad. Sci. Paris 280 (1975), 365-367.

[2 ] M. Buchner and W. Kucharz, Topological triviality of a family of zero-sets, Proc. Amer. Math. Soc. 102 (1988), 699-705.

[3] M. Coste and M. Shiota, Thom's first isotopy lemma : a semialgebraic version with uniform bounds (to appear).

[4] T. Fukui, Toric resolution and real function, preprint.

[ 5 ] H. King, Topological type in families of germs, Invent. Math. 62 (1980), 1-13.

[6] S. Koike, On strong $C^{0}$-equivalence of real analytic functions, J. Math. Soc. Japan 45 (1993), 313-320.

[ 7 ] S. Koike, Strong $C^{0}$-triviality of family of weighted homogeneous polynomials, (in preparation).

[8] T.C. Kuo, On $C^{0}$-sufficiency of jets of potential functions, Topology 8 (1969), 167-171.

[9] T.C. Kuo, Characterizations of v-sufficiency of jets, Topology 11 (1972), 115-131.

[10] B. Malgrange, Ideals of differentiable functions, Oxford Univ. Press, 1966.

[11] M. Shiota, Classification of Nash manifolds, Ann. Inst. Fourier 33 (1983), 209-232

[12] M. Shiota, Nash manifolds, Lect. Notes in Math. 1269, Springer, 1987.

DEPARTMENT OF MATHEMATics

HYOGO UNIVERSITY OF TEACHER EDUCATION

YASHIRO-CHO, KATO-GUN

HYOGO $673-14$, JAPAN 